\title{
Radial Profile Measurements of Electron Temperature and Density Using the Thomson Scattering System in GAMMA 10/PDX ${ }^{*}$
}

\author{
Masayuki YOSHIKAWA, Junko KOHAGURA, Masayuki CHIKATSU, Yoriko SHIMA, \\ Mizuki SAKAMOTO, Yousuke NAKASHIMA, Ryutaro MINAMI, Ichihiro YAMADA ${ }^{1)}$, \\ Ryo YASUHARA $^{1)}$, Hisamichi FUNABA ${ }^{1)}$, Takashi MINAMI') and Naoki KENMOCHI ${ }^{3)}$ \\ Plasma Research Center, University of Tsukuba, 1-1-1 Tennodai, Tsukuba, Ibaraki 305-8577, Japan \\ ${ }^{1)}$ National Institute for Fusion Science, 322-6 Oroshi-cho, Toki, Gifu 509-5292, Japan \\ ${ }^{2)}$ Institute of Advanced Energy, Kyoto University, Gokasho, Uji, Kyoto 611-0011, Japan \\ ${ }^{3)}$ Graduate School of Frontier Sciences, University of Tokyo, Kashiwa, Chiba 277-8561, Japan
}

(Received 21 December 2017 / Accepted 28 March 2018)

\begin{abstract}
We developed the YAG Thomson scattering (TS) system for electron temperature and density radial profiles in a single laser shot in the tandem mirror GAMMA 10/PDX. The optical collection system for the TS light is constructed using spherical mirrors and nine bundled optical fibers. The measurable radial positions are 5-cm intervals in a region of $\pm 20 \mathrm{~cm}$ due to the fixed position of the bundled optical fibers, which are set on a fixedheight optical platform. We replaced this with a lab jack to make the bundled optical fiber positions movable. Consequently, we can measure the radial profiles of the electron temperature in more detail. Moreover, we added a third spherical light collection mirror at the bottom of the main spherical collection mirror to increase the TS light intensity in the edge plasma region. In this way, we can obtain a slightly larger TS signal intensity than in the case without a third collection mirror. The radial electron temperatures and densities at 15 radial positions in GAMMA 10/PDX are successfully obtained. For the in-situ calibration of the electron density measurements of the TS system, we compare the TS system measurements to the electron line density measured using a microwave interferometer system.
\end{abstract}

(c) 2018 The Japan Society of Plasma Science and Nuclear Fusion Research

Keywords: Thomson scattering, radial profile, electron temperature, electron density, tandem mirror GAMMA 10/PDX

DOI: $10.1585 /$ prr.13.3402051

\section{Introduction}

Thomson scattering (TS) is the most useful diagnostic available to measure the electron temperature and density in fusion devices [1-10]. To apply a TS system in lower electron density plasmas, such as GAMMA 10/PDX and the spherical region of fusion plasmas, large numerical aperture collection optics and large scattering lengths are required. In GAMMA 10/PDX, we installed an yttriumaluminum-garnet (YAG)-TS system with large angle collection optics and a large scattering length optical system $[8,9]$. The YAG-TS system can measure radial electron temperatures and densities at seven fixed positions in a single laser shot at $10 \mathrm{~Hz}$. GAMMA $10 / \mathrm{PDX}$ is effectively an axisymmetric minimum-B anchored tandem mirror with a thermal barrier at both end-mirrors [11]. The $x$ and $y$-axes are perpendicular to the magnetic field in the vertical and horizontal directions, respectively. The $z$-axis is parallel to the magnetic field. The plasma is created by plasma guns and heated and sustained using ion cyclotron heating (ICH) systems. In addition to ICH, electron cy-

author'se-mail: yosikawa@prc.tsukuba.ac.jp

*) This article is based on the presentation at the 26th International Toki Conference (ITC26). clotron heating $(\mathrm{ECH})$ is applied to produce the electron and ion confinement potential in the plug $(\mathrm{P})$ and barrier (B) cells. In the central cell, we performed direct electron heating by applying the central (C) ECH [12]. Super molecular beam injection (SMBI) experiments have been performed in the central cell to increase the plasma density and to create the higher end loss flux for divertor plasma experiments [13]. In the end region, we installed a divertor simulation experimental module to perform the divertor simulation experiments. The electron density, electron temperature, and ion temperature are measured using the YAG-TS system, microwave interferometers, a soft X-ray measurement system, and a charge exchange neutral particle analyzer system [14-17]. The typical electron density and electron and ion temperatures are approximately $2 \times$ $10^{12} \mathrm{~cm}^{-3}, 100 \mathrm{eV}$, and $5 \mathrm{keV}$, respectively, during the application of $\mathrm{P} / \mathrm{B}-\mathrm{ECH}$.

The optical collection system for the TS light is constructed using spherical mirrors and nine bundled optical fibers. The measurable radial positions are $5-\mathrm{cm}$ intervals in the region of $\pm 20 \mathrm{~cm}$ due to the fixed position of the bundled optical fibers, which are set on a fixed-height optical platform. The e-folding radius of the C-ECH power 
density of the radiation distribution on the resonance surface is approximately $3 \mathrm{~cm}$, and the FWHM of the $x$-axis $\mathrm{H} \alpha$ emission radial profile on the SMBI experiment is approximately $6 \mathrm{~cm}$ just after the injection to the plasma. To measure the radial TS signals in more detail, less than a 5-cm interval, we replaced the fixed-height optical platform with a lab jack to make the bundled optical fiber position movable. Moreover, we added a third spherical light collection mirror at the bottom of the main spherical collection mirror to increase the TS light intensity in the upper side edge plasma region. These improvements will be useful for studying the heating mechanisms of the C-ECH and SMBI experiments. During the GAMMA 10/PDX plasma experiments, we sometimes need to change the optical components after performing the density calibration gas scattering experiments due to damage to the optical components or to reset the optical systems to improve the scattering signal intensity. For the in-situ calibration of the electron density measurements of the TS system, we compare the measurements to the electron line density measured by the 6-channel microwave interferometer (MIF) system $[16,17]$.

In this paper, we demonstrate the improved YAG-TS system for measuring the detailed radial profiles of electron temperatures and densities with the newly installed lab jack system and third additional collection mirror after performing in-situ calibration using the microwave interferometer system in the central cell of GAMMA 10/PDX.

\section{Experimental Apparatus}

The YAG-TS system installed at $z=60 \mathrm{~cm}$ in the GAMMA 10/PDX central cell is shown in Fig. 1. The YAG-TS system is composed of a laser, incident optics, light collection optics, signal detection electronics, and a data recording system. A $10-\mathrm{Hz} \mathrm{Nd:YAG} \mathrm{laser} \mathrm{(Contin-}$ uum, Powerlite 9010) with an energy per pulse of $2 \mathrm{~J}$ and a pulse width of approximately $10 \mathrm{~ns}$ operating at the fundamental wavelength of $1064 \mathrm{~nm}$ is used. The laser goes

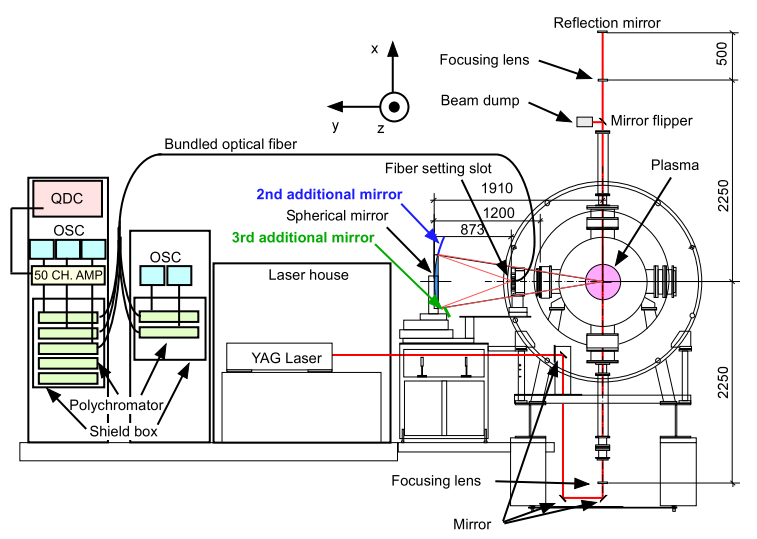

Fig. 1 Side view of the TS system in the GAMMA 10 central cell. through a short pass half mirror, two Faraday rotators, two polarizers, two $\lambda / 2$ plates, four mirrors, and an iris on the optical bench. The laser beam is injected into the plasma from the down side port though a focusing lens of $f=$ $2.25 \mathrm{~m}$. The laser beam diameter at the plasma center is approximately $1 \mathrm{~mm}$. The polarization of the laser in the plasma is in the $z$-direction. The light collection optics were optimized with the help of optical system design software (ZEMAX). For the light collection optics, we used a spherical mirror with an $\mathrm{Al}: \mathrm{SiO}_{2}$ coating, a curvature radius of $1.2 \mathrm{~m}$, and a diameter of $0.6 \mathrm{~m}$. For the down side edge plasma light collection, we set a second spherical mirror with a curvature radius of $1.2 \mathrm{~m}$ and a diameter of $0.2 \mathrm{~m}$ on the top of the main spherical mirror [10]. The additional mirror system is useful for increasing the TS signal from the edge plasma in the TS system. Moreover, we added a third collection mirror with a curvature radius of $1.0 \mathrm{~m}$ and a rectangular size of $0.12 \mathrm{~m} \times 0.30 \mathrm{~m}$ set at the down side of the main spherical mirror to double the TS light from the upper side edge plasma region. The distance between the main mirror center and the additional mirror center is approximately $0.34 \mathrm{~m}$. The mirror size is optimal for obtaining the maximum collection efficiency, considering the solid angle and the setting position. The scattered light is collected by the spherical mirrors, reflected, and reaches a bundled optical fiber with a cross section of $2 \mathrm{~mm} \times 7 \mathrm{~mm}$. The magnification of the collection optics is $1 / 2.2$. The length of the scattering volume along the laser is $15.4 \mathrm{~mm}$, and the scattering angle is $90^{\circ}$. A solid angle of $0.078 \mathrm{sr}$ can be realized by the light collection optics of the main collection mirror. After passing through the plasma, the laser beam is ejected from the upper side port and absorbed by the beam dump through the mirror flipper system. The 6.67-m-long bundled optical fiber (Mitsubishi Densen, FS10-43001A) is connected to a 5-channel polychromator. The bundled optical fiber has a very large numerical aperture of approximately 0.47 . The fiber aperture should be located approximately $0.873 \mathrm{~m}$ from the spherical mirror. The fiber positions were fixed by the fiber slot on the fixed-high optical platform. The measurable radial positions are $5-\mathrm{cm}$ intervals in the region of $\pm 20 \mathrm{~cm}$. To measure the radial TS signals in intervals of less than $5 \mathrm{~cm}$, we replaced the fixed-height optical platform with a lab jack (Thorlabs, L490) to make the bundled optical fiber position movable. The polychromator is composed of five relay and collection lenses, five interference filters, and five silicon avalanche photodiodes (Si-APD, PerkinElmer, C30950E and C30659-1060-3AH). Four-channel high-speed oscilloscopes (IWATSU, DS5524, and DS5524A) were used to measure four wavelength channels simultaneously with a bandwidth of $200 \mathrm{MHz}$ and a sampling rate of $1 \mathrm{GS} / \mathrm{s}$ at $10 \mathrm{~Hz}$. The measured signals are recorded by a Windows PC with LabVIEW analyzing software. We conducted wavelength calibration experiments of the spectral sensitivities of the 5-channel polychromators using a standard 


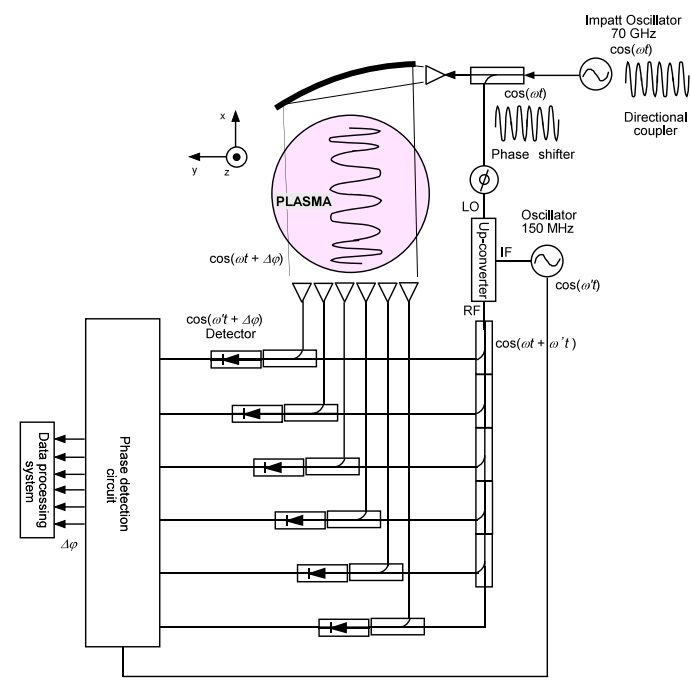

Fig. 2 Schematic view of the6-channel MIF system.

light (Oriel, \#66890) and a spectrometer (Oriel, CS130). In the GAMMA 10/PDX-YAG-TS system, the electron temperature is deduced via a nonlinear least squares fit procedure at the integrated output signals of each channel. The fit is obtained using a look-up table, which contains the calculated intensities expected at each channel for $1-\mathrm{eV}$ intervals up to $500 \mathrm{eV}$. By interpolating between the tabulated values, the values of the electron temperature that minimize the chi-squared value are obtained.

We performed the in-situ density calibration of the TS system using the 6-channel 70-GHz MIF system (Fig. 2), which is set at $z=0 \mathrm{~cm}$ for electron line density radial profile measurements in a single plasma shot. The interferometer was designed based on the principles of Gaussianbeam propagation theory and ray-tracing code. The system was configured as a heterodyne interferometer consisting of a $70-\mathrm{GHz}(1 \mathrm{~W})$ IMPATT oscillator and a $150-\mathrm{MHz}$ oscillator. The probe microwave beam is injected into the plasma from the emission horn after being reflected by a spherical mirror into a sheet beam. The beam is received by six receiver horns mounted at the bottom outside the GAMMA 10 port. We can obtain the electron density radial profiles in the $y$-direction using the Abel transform technique.

\section{Calibration Experiments}

Raman and Rayleigh calibration experiments were performed to set the optical system and the stray light in the evaluation of the GAMMA 10/PDX-YAG-TS system. Nitrogen gas was used, and the pressure in the GAMMA 10 device was raised to 300 Torr. The measured scattering signal is proportional to the gas pressure. The linear component indicates the scattering light, and the offset indicates the stray light. The Raman scattering calibration experiments for the radial position measurements of $x=0 \mathrm{~cm}$, $\pm 5 \mathrm{~cm}, \pm 10 \mathrm{~cm}, \pm 15 \mathrm{~cm}$, and $\pm 20 \mathrm{~cm}$, were performed si-

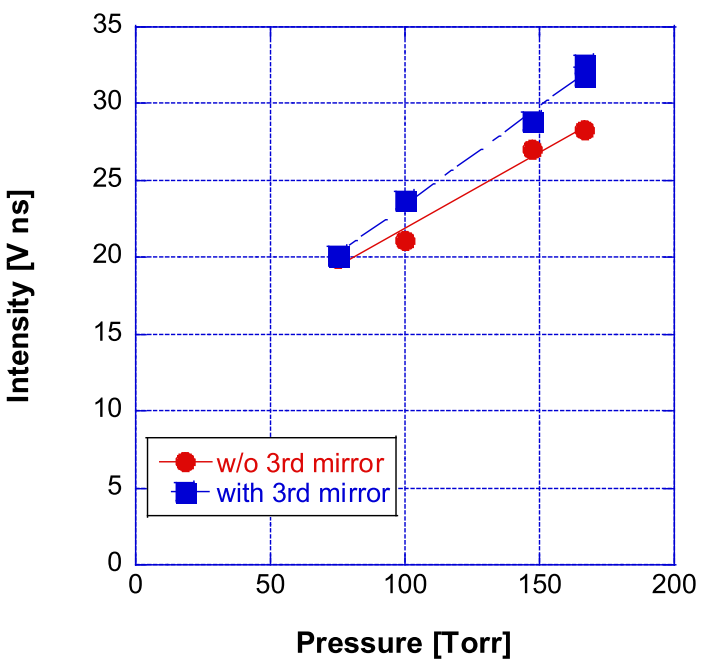

Fig. 3 The integrated Raman scattering intensities versus the gas pressures without and with the third mirror at $x=20 \mathrm{~cm}$.

multaneously. Then, we obtained the electron density by comparing the linear components of the Rayleigh and Raman scattering experiments for each measuring position to the integrated TS signal intensity. The estimated increase of the signal intensities with the third mirror are approximately $20 \%, 50 \%$, and $70 \%$, at radial positions of $x=$ $10 \mathrm{~cm}, 15 \mathrm{~cm}$, and $20 \mathrm{~cm}$, respectively. In Fig. 3, we show the effect of increasing the scattering signal intensity by using the third mirror at $x=20 \mathrm{~cm}$. The closed red circles and the blue squares show the intensities without and with the additional third mirror, respectively. The increases in the signal intensities with the third mirror at $x=10 \mathrm{~cm}$, $15 \mathrm{~cm}$, and $20 \mathrm{~cm}$ are approximately $5 \%, 10 \%$, and $12 \%$, respectively. The stray light in the core plasma region is less than approximately $10 \%$. The stray light in the outer region is not small. However, we calculated the electron temperature after subtracting the stray light intensity and the background noise from the measured signal taking into consideration the stray light before and after the plasma discharge. Then, we measured the electron temperature and density under this stray light condition. Unfortunately, the increases in the signal intensities due to the additional third mirror are very small compared to the estimated values. We need to further optimize the position of the third mirror and its tilt angle setting to increase the signal intensities to the estimated values.

\section{Radial Electron Temperature and Density}

The plasma was produced from $t=50 \mathrm{~ms}$ to $t=$ $240 \mathrm{~ms}$ and was heated by the ICH from $t=51 \mathrm{~ms}$ to $t$ $=240 \mathrm{~ms}$. In Fig. 4, we show the integrated line densities calculated by the electron density measured using the TS system (red closed circles) and the measured line densities at $x=0 \mathrm{~cm}$ measured using the MIF system (blue closed 


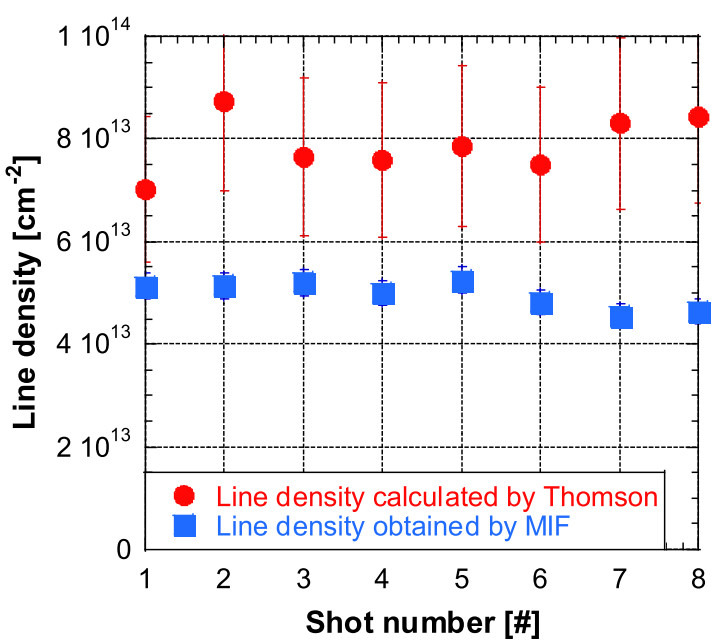

Fig. 4 Comparison of the line densities measured using the MIF and TS systems.

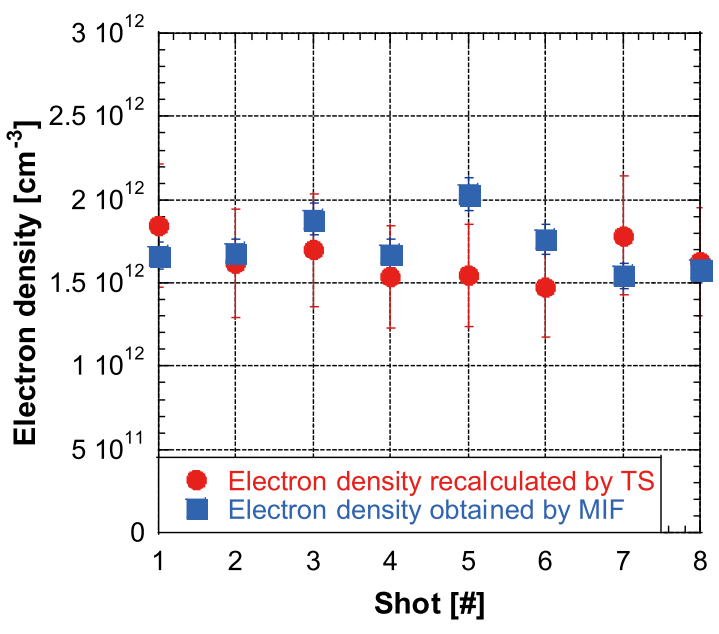

Fig. 5 Comparison of the electron densities obtained using the MIF and in-situ calibrated TS systems.

squares) in the same eight plasma shots. Every electron line density in Fig. 4 was obtained in a single plasma shot by the TS and 6-channel MIF systems. The averaged ratio of the integrated line densities obtained by the TS system and those obtained by the MIF system is approximately 1.6. In Fig. 5, we show the electron densities obtained by the 6-channel MIF system (blue closed squares) and the in-situ calibrated TS system (red closed circles) divided by the above ratio. After the calibration, the TS measured electron density is the same as that calculated by the 6channel MIF system within an error of $20 \%$ for every shot. We can compare the measurements shot-by-shot to check the total sensitivity of the TS system. The calibration coefficients changed a little after some system adjustments of the TS system and the YAG laser intensity.

Figures 6 (a) and 6 (b) show the radial electron temperature and density profiles measured using the TS system at $t=155.1 \mathrm{~ms}$ when changing the measuring positions by

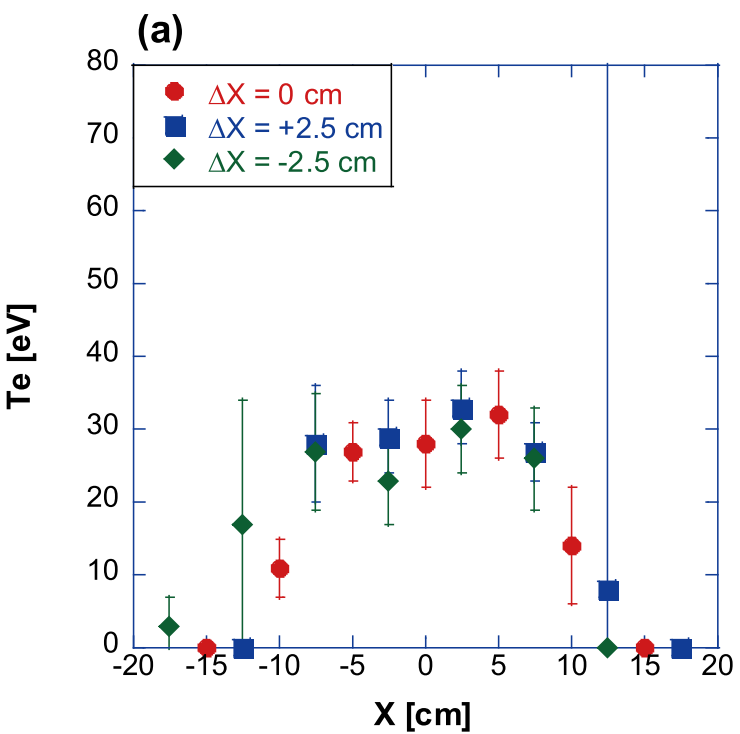

(b)

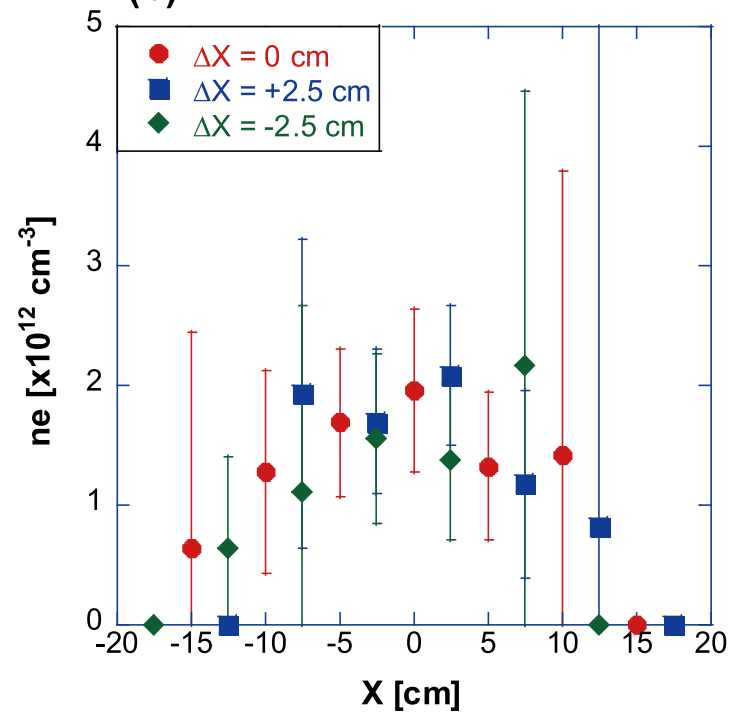

Fig. 6 Radial profiles of (a) the electron temperature and (b) the density when changing the lab jack position.

$\pm 2.5 \mathrm{~cm}$ from the base position of the lab jack shot-by-shot in three plasma shots, respectively. Here, the red closed circles, blue closed squares, and green diamonds show the results at $\Delta X=0 \mathrm{~cm},+2.5 \mathrm{~cm}$, and $-2.5 \mathrm{~cm}$ from the base position of the lab jack, respectively. Then, we measured the radial positions of the electron temperature and density in 2.5 -cm intervals.

\section{Summary}

We added a third collection mirror to improve the TS signals from the edge region plasma; this increased the TS signal intensity slightly; however, we need to further optimize the mirror setting. The detailed radial electron temperature and density profiles were successfully measured using the newly installed lab jack system. In addition, we used an in-situ calibration comparing the mea- 
sured line density using the microwave interferometer system and calculated the line density using the electron densities from the YAG-TS system.

\section{Acknowledgments}

The authors thank the members of the GAMMA 10/PDX group of the University of Tsukuba for their collaboration. This study was conducted with the support and under the auspices of the NIFS Collaborative Research Program (NIFS11KUGM056) and the Bidirectional Collaboration Research Programs (NIFS14KUGM086, NIFS14KUGM088).

[1] K. Narihara et al., Fusion Eng. Des. 34-35, 67 (1997).

[2] J.H. Foote et al., Rev. Sci. Instrum. 61, 2861 (1990).

[3] J. Hatae et al., Rev. Sci. Instrum. 70, 772 (1999).

[4] T. Hatae et al., Rev. Sci. Instrum. 77, 10E508 (2006).
[5] J. Hiratsuka et al., Plasma Fusion Res. 5, 044 (2010).

[6] H. Togashi et al., Plasma Fusion Res. 9, 1202005 (2014).

[7] M. Yu Kantor et al., Plasma Phys. Control. Fusion 51, 055002 (2009).

[8] M. Yoshikawa et al., Plasma Fusion Res. 6, 1202095 (2011).

[9] M. Yoshikawa et al., J. Instrum. 8, C10016 (2013).

[10] M. Yoshikawa et al., J. Instrum. 10, C11006 (2015).

[11] Y. Nakashima et al., Nucl. Fusion 57, 116033 (2017).

[12] R. Minami et al., Trans. Fusion Sci. Technol. 59, 244 (2011).

[13] Md. M. Islam et al., Plasma Fusion Res. 11, 2402053 (2016).

[14] M. Yoshikawa et al., Plasma Fusion Res. 8, 1205169 (2013).

[15] M. Yoshikawa et al., Plasma Fusion Res. 9, 1202126 (2014).

[16] M. Yoshikawa et al., Rev. Sci. Instrum. 79, 10E706 (2008).

[17] Y. Hasegawa et al., Trans. Fusion Sci. Technol. 63, 337 (2013). 